\title{
Multiple Reaction Pathways in the Morphinone Reductase Catalyzed Hydride Transfer Reaction
}

Xi Chen, Steven D. Schwartz*

Department of Chemistry and Biochemistry, University of Arizona, Tucson, Arizona, 1306 East University Boulevard, Tucson, Arizona 85721, United States

\section{Corresponding Author}

Steven D. Schwartz

*sschwartz@email.arizona.edu

Department of Chemistry and Biochemistry, University of Arizona, Tucson, Arizona, 1306 East University Boulevard, Tucson, Arizona 85721, United States 


\section{Table of contents:}

SI Figure 1: Illustration of the magnitude of reduction used as GMM inputs

SI Figure 2: Relative positions of residues in the reaction coordinate of the wild type MR pathway 1

SI Figure 3: Important conformation change process along the reaction progress

SI Figure 4: Overlay of the FMN isoalloxazine plane geometry prior to its distortion of all five reaction pathways

SI Figure 5: Probability density distribution of the three structure features in all five reaction pathways

SI Figure 6: Active site geometry with a broken Asn189-NADH nicotinamide hydrogen bond 


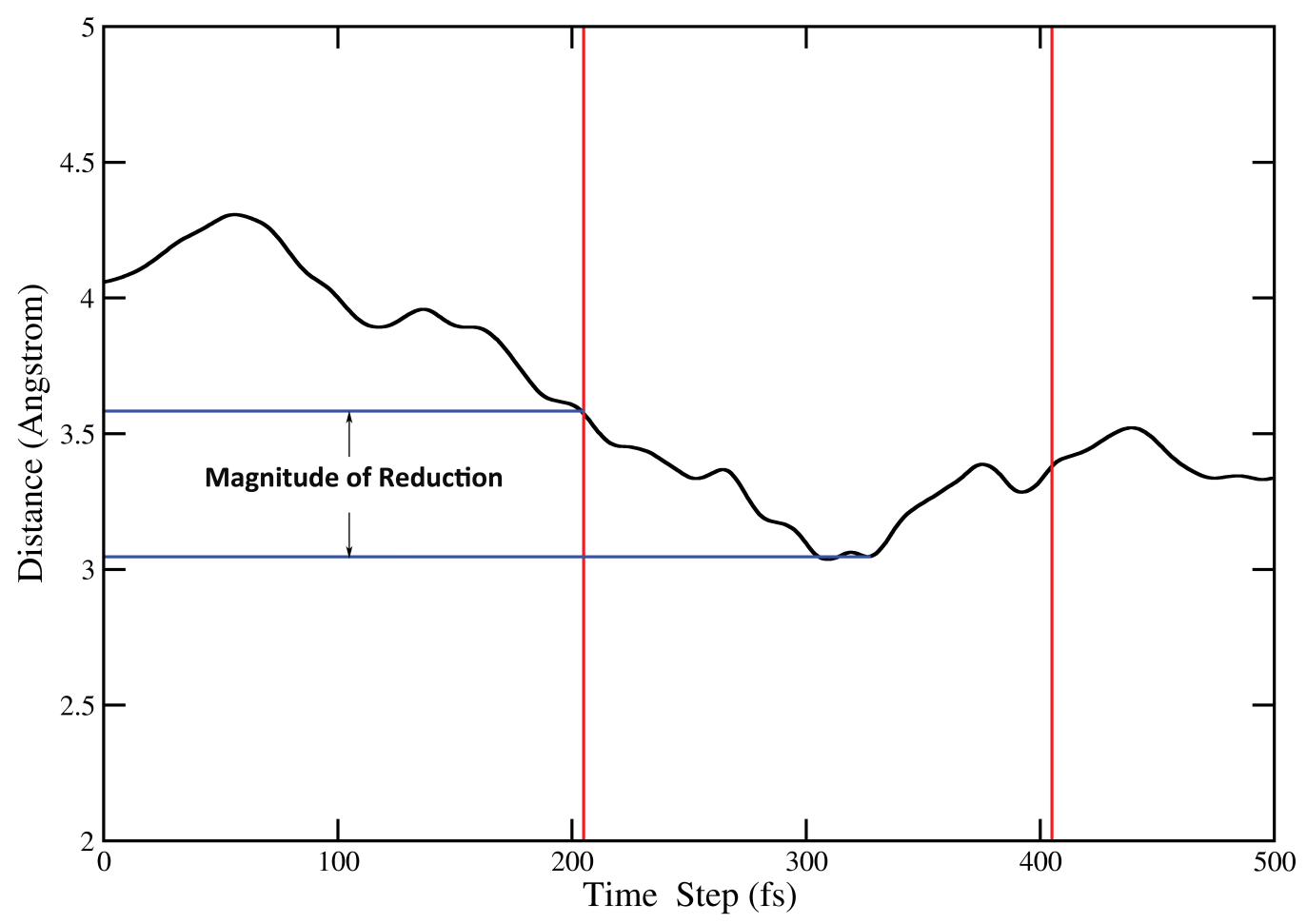

SI Figure 1. Example of the 'magnitude of reduction' definition. Black solid line shows the time evolution of the NADH ribose ring - NADH nicotinamide ring distance taken from an example trajectory. The 200fs window directly prior to the transition state is labeled by the two vertical red solid lines. The maximum and the minimum of the distance with in the $200 \mathrm{fs}$ window are labeled by the two blue horizontal lines. Absolute values of the difference between the maximum and the minimum is used as the definition of the magnitude of reduction. 


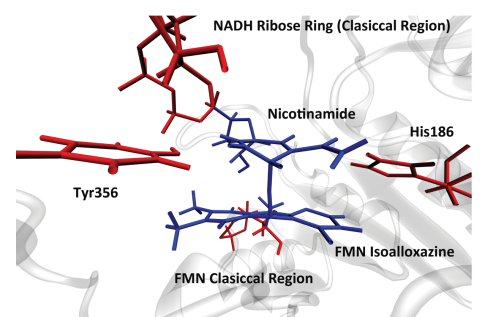

(a)

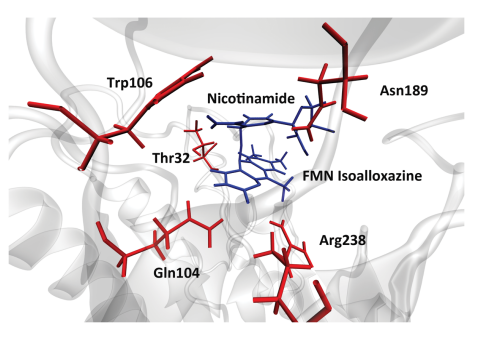

(b)

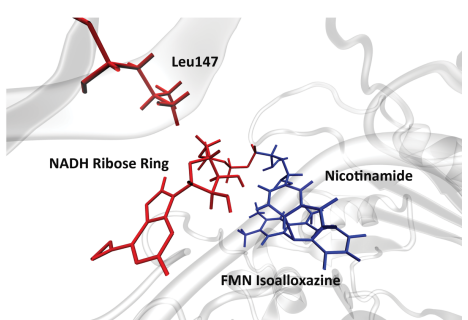

(c)

SI Figure 2. Relative positions of degrees of freedom involved in the reaction coordinate of the wild type MR pathway 1. In all three figures, QM region is colored in blue, while protein residues, the NADH classical region and the FMN classical region are colored in red. SI Figure 2(a) shows relative positions of Tyr356, His186, the NADH classical region (specifically the ribose ring), the FMN classical region. SI Figure 2(b) shows the relative position of Thr32, Gln104, Trp106, Asn189, Arg238. SI Figure (c) shows the relative position of Leu147 to the NADH ribose ring. 


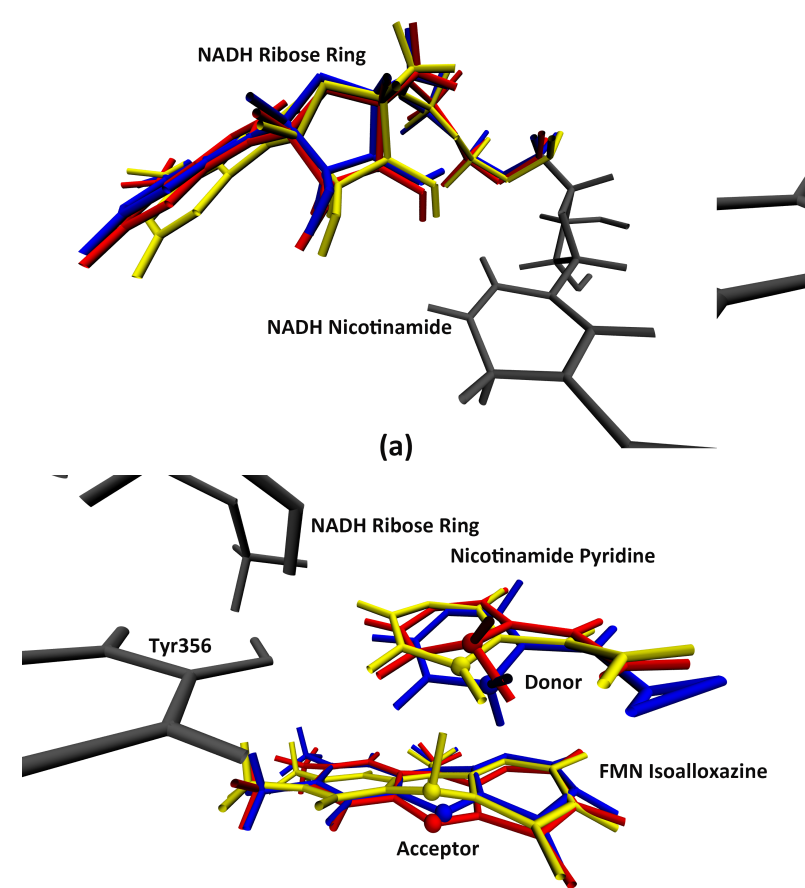

(c)

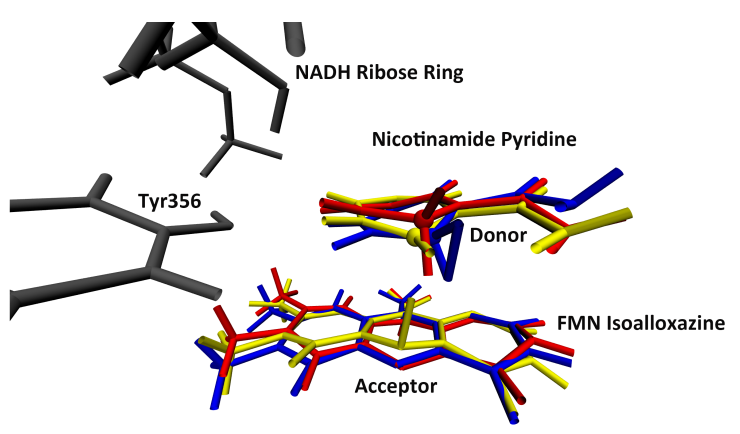

(b)

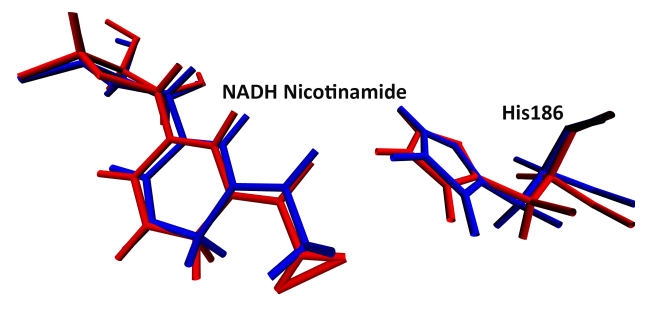

(d)

SI Figure 3. Conformation change process of the MR active site. SI Figure 3(a) shows the overlay of three structures focusing on the NADH ribose ring, taken at $300 \mathrm{fs}$ prior to the transition state (blue), 100fs prior to the transition state (red) and the transition state (yellow) from the wild type MR pathway 1. The NADH pyridine ring is colored grey. SI Figure 3(b) shows the conformational change process of the wild type pathway 2, looking from the front of the active site. Three structures taken at $300 \mathrm{fs}$ prior to the transition state (blue), 200fs prior to the transition state (red) and the transition state (yellow) are overlaid. SI Figure 3(c) shows the conformational change process of the N189A pathway 1, looking from the front of the active site. Three structures taken at $400 \mathrm{fs}$ prior to the transition state (blue), 200fs prior to the transition state (red) and the transition state (yellow) are overlaid. SI Figure 3(d) shows the overlay of two structures looking from the top of the NADH nicotinamide plane, taken at 300fs prior to the transition state (blue) and at the transition state (red) taken from the N189 pathway 2 . 

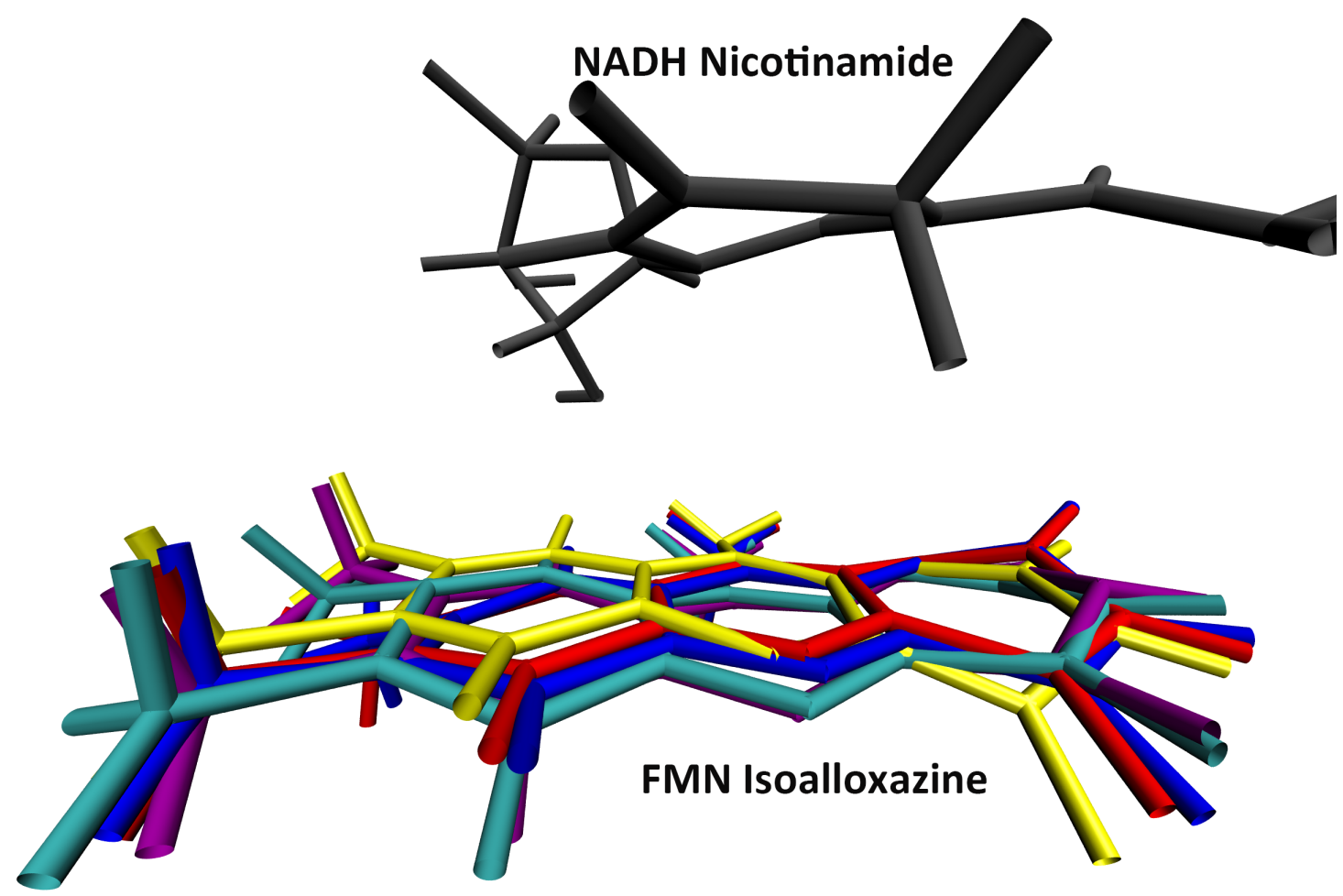

SI Figure 4. FMN isoalloxazine geometry prior to the reaction. SI Figure 4 shows the active site geometry prior to the FMN isoalloxazine distortion in TPS trajectories, focusing on the FMN isoalloxazine plane. Structures from the wild type path 1 (cyan), wilt type pathway 2 (purple), N189A pathway 1 (blue), N189A pathway 2 (red) and N189A pathway 3 (yellow) are overlaid. Relative position of the NADH nicotinamide is colored in gray. 

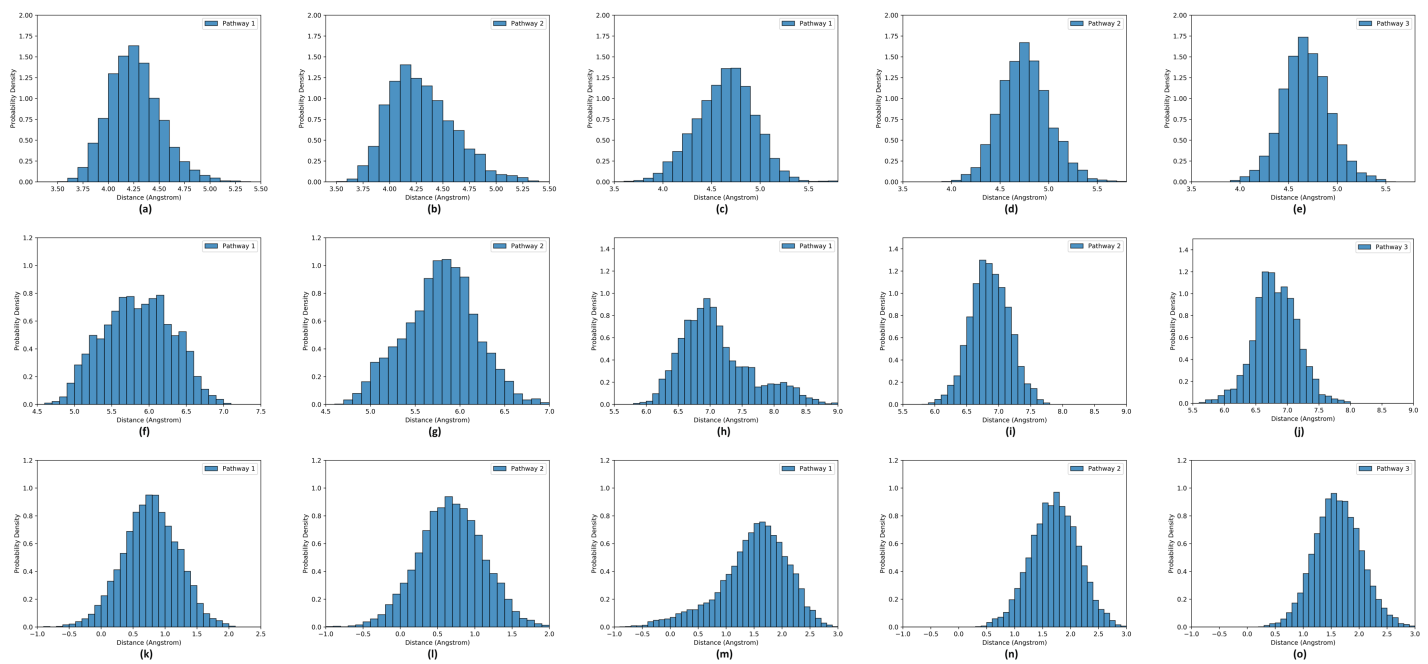

SI Figure 5. Probability density distributions of three structural features from five reaction pathways. In SI Figure 5, in each row, from left to right is the probability density distribution of the wild type pathway 1 , wild type pathway 2, N189A pathway 1, N189A pathway 2, N189A pathway 3 . The first row shows the probability density distribution of the NADH pyridine ringFMN isoalloxazine distance; the second row shows the probability density distribution of the NADH ribose ring-NADH pyridine ring distance; the third row shows the probability density distribution of the difference between the N1-N10 distance and the C4-N5 distance. 


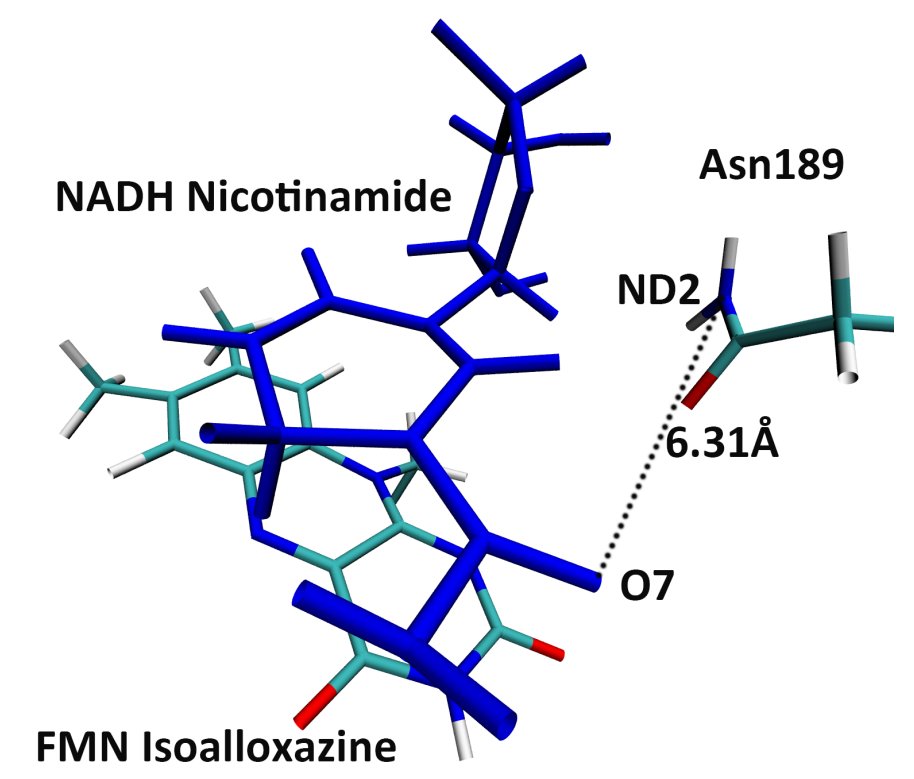

SI Figure 6. Active site geometry with a broken Asn189-NADH nicotinamide hydrogen bond. SI Figure 6 shows a structure from the wild type reactant states in which the Asn189 nicotinamide $\mathrm{O} 7$ hydrogen bond is broken, but the nicotinamide plane is still parallel to the FMN isoalloxazine. The O7-Asn189 ND2 distance is marked by the black dashed line. 